\title{
The construction of solutions to
}

\section{Zakharov-Kuznetsov equation with fractional power nonlinear terms}

Yang Liu' ${ }^{1 *}$ and Xin Wang

*Correspondence:

liuyangdqpi@126.com

${ }^{1}$ College of Petroleum Engineering,

Northeast Petroleum University,

Daqing, China

\section{包 Springer}

\begin{abstract}
In the paper, we study a plasma fluid physical model, namely the Zakharov-Kuznetsov (ZK, for simplicity) equation with fractional power nonlinear terms by the complete discrimination system for polynomial method, and give a detailed construction of all its single traveling wave solutions. The results show abundant traveling wave patterns of the ZK equation.
\end{abstract}

Keywords: The complete discrimination system for polynomial; Exact solution; Traveling wave solution; Zakharov-Kuznetsov equation

\section{Introduction}

Due to the role of nonlinear differential equations in modeling physical phenomena, an important task in nonlinear science is to find the exact solutions to those equations. Correspondingly, a lot of useful methods have been proposed to solve nonlinear differential equations, including direct expansion methods and ansatz methods (see, for example [15] and the references therein). In addition, some further arguments on L-integrability and Liouville theorem in infinite-dimensional Hamiltonian systems have been given [6]. However, for those direct expansion methods, a weakness is that one cannot obtain complete results though the considered equations can be reduced to integral forms. In order to overcome this weakness, Liu proposed the complete discrimination system for polynomial method to give the complete classifications of all single traveling wave solutions for such nonlinear differential equations [7-11]. In Liu's method, we firstly reduce the considered partial differential equation to the integral form under the traveling wave transformation, then we can use the complete discrimination system for polynomial to give the factorization when the integrand is determined by a polynomial, and finally we classify the solutions of the corresponding integral. If the equation cannot be directly reduced to an integral form, we can use Liu's trial equation method to get an integrable sub-equation, from which we obtain exact solutions by using the complete discrimination system for polynomial [12-17]. Liu's methods have been further applied and developed extensively to solve more nonlinear problems (see, for example, [17-23] and the references therein). For exact solutions of nonlinear differential equations, there are a large number of results (see, for example, Seadawy et al.s interesting works [24-33]). Recently, Ma et al. proposed a

(c) The Author(s) 2019. This article is distributed under the terms of the Creative Commons Attribution 4.0 International License (http://creativecommons.org/licenses/by/4.0/), which permits unrestricted use, distribution, and reproduction in any medium, provided you give appropriate credit to the original author(s) and the source, provide a link to the Creative Commons license, and indicate if changes were made. 
powerful method, namely the transformed rational function method, for generating traveling wave solutions and studied deeply and extensively another kind of exact solutions called lump solutions [34-41].

In the paper, we consider a kind of the Zakharov-Kuznetsov (ZK, for simplicity) equations with fractional power nonlinear terms. The ZK equation was first derived to describe the behavior of weakly nonlinear ion-acoustic waves in plasma which comprises cold ions and hot isothermal electrons when there is a uniform magnetic field [42-44]. Moreover, it also governs two-dimensional modulations of a KdV soliton equation in fluid mechanics. The usual ZK equation reads

$$
u_{t}+a u u_{x}+\left(u_{x x}+u_{y y}\right)_{x}=0
$$

which is more difficult to study than the KP equation because it is not integrable under the meaning of the inverse scattering transform method. In addition, we know that the solitary-wave solutions of the ZK equation are inelastic.

Furthermore, to describe ion-acoustic waves in cold-ion plasma when the behavior of electrons is not isothermal, Schamel [45] derived the new ZK equations with fractional power nonlinear terms as follows:

$$
u_{t}+u^{\frac{1}{2}} u_{x}+a u_{x x x}=0
$$

and

$$
u_{t}+\left(1+b u^{\frac{1}{2}}\right) u_{x}+\frac{1}{2} u_{x x x}=0
$$

Monro and Parkes also obtained a different fractional power form of the ZK equation

$$
16 u_{t}+20\left(u^{\frac{3}{2}}\right)_{x}+u_{x x x}=0
$$

Wazwaz obtained some special forms of exact solutions to a fractional ZK equation by the sine-cosine ansatz method [46]. Other related studies on the equation can be seen in [47, 48]. However, to our knowledge, all single traveling wave solutions to the ZK equations have not been given [43-48].

In the paper, we use the complete discrimination system for polynomial to give a complete classification of all single traveling wave solutions for two fractional power ZK equations. From a practical point of view, the classification of traveling wave solutions is very convenient since we can determine the type of solutions when the conditions of concrete parameters are given.

The paper is organized as follows. In Sect. 2, we give the classification for $\gamma=\frac{1}{2}$. In Sect. 3, we give the classification for $\gamma=\frac{3}{2}$. In the last section, we give a short discussion and conclusion.

\section{Classification of solutions: first case}

We consider a general form of the fractional power ZK equation

$$
a u_{t}+b\left(u^{\gamma}\right)_{x}+u_{x x x}=0,
$$


where $a$ and $b$ are coefficients, and $\gamma$ in general is a fractional number such as $\gamma=\frac{1}{2}$ and $\gamma=\frac{3}{2}$ and so on. It is easy to see that this ZK equation includes the previous ZK equations as special examples.

Substituting traveling wave transformation

$$
u(x, t)=u(\xi), \quad \xi=x-\omega t
$$

into Eq. (5), we get the ODE as follows:

$$
-a \omega u^{\prime}+b\left(u^{\gamma}\right)^{\prime}+u^{\prime \prime \prime}=0 .
$$

Integrating the above equation twice yields

$$
\left(u^{\prime}\right)^{2}=b_{0}+b_{1} u+a \omega u^{2}-\frac{2 b}{\gamma+1} u^{\gamma+1},
$$

where $b_{0}$ and $b_{1}$ are two integral constants. If $\gamma$ is a rational number, we can write it as $\gamma=\frac{n}{m}$, and then we have

$$
\left(u^{\prime}\right)^{2}=b_{0}+b_{1} u+a \omega u^{2}-\frac{2 b m}{n+m} u^{\frac{n+m}{m}} .
$$

Further, we take $u=v^{m}$ to get

$$
m^{2} v^{2 m-2}\left(v^{\prime}\right)^{2}=b_{0}+b_{1} v^{m}+a \omega v^{2 m}-\frac{2 b m}{n+m} v^{n+m} .
$$

Writing it as an integral form, we have

$$
\int \frac{v^{m-1} \mathrm{~d} v}{\sqrt{b_{0}+b_{1} v^{m}+a \omega v^{2 m}-\frac{2 b m}{n+m} v^{n+m}}}= \pm \frac{1}{m}\left(\xi-\xi_{0}\right),
$$

where $\xi_{0}$ is an integral constant. This integral form is our starting point of solving exact solutions. If we denote $F(v)=b_{0}+b_{1} v^{m}+a \omega v^{2 m}-\frac{2 b m}{n+m} v^{n+m}$, it is easy to see that the solutions of integral depend on the roots of $F(v)$ completely. A powerful mathematical tool, namely a complete discrimination system for polynomial, can be used to solve the problem. Firstly, we take $\gamma=\frac{1}{2}$ respectively to give all solutions to the corresponding integral

$$
\int \frac{v \mathrm{~d} v}{\sqrt{b_{0}+b_{1} v^{2}-\frac{4 b}{3} v^{3}+a \omega v^{4}}}= \pm \frac{1}{2}\left(\xi-\xi_{0}\right) .
$$

We consider two cases $b_{0}=0$ and $b_{0} \neq 0$ to give solutions respectively.

Case 1. $b_{0}=0$. Then the integral form becomes

$$
\int \frac{\mathrm{d} v}{\sqrt{b_{1}-\frac{4 b}{3} v+a \omega v^{2}}}= \pm \frac{1}{2}\left(\xi-\xi_{0}\right) .
$$

Denote $\Delta=b_{3}^{2}-4 b_{1} a \omega$ as the discrimination of $f(v)=b_{1}-\frac{4 b}{3} v+a \omega v^{2}$. According to the values of $\Delta$, we obtain the following solutions. 
Family 2.1.1. $\Delta=0$. Then we have $f(v)=a \omega(v-\alpha)^{2}$, where $\alpha=\sqrt{\frac{b_{1}}{a \omega}}$. We get the solution

$$
u=\left\{\alpha \pm \exp \left( \pm \frac{\sqrt{a \omega}}{2}\left(\xi-\xi_{0}\right)\right)\right\}^{2}
$$

Family 2.1.2. $\Delta>0$. Then we have $f(v)=a \omega(v-\alpha)(v-\beta)$, where $\alpha$ and $\beta$ are two distinct real roots of $f=0$. We get the solution

$$
u=\left\{\frac{\alpha-\beta\left(1 \pm \exp \left( \pm \frac{\sqrt{a \omega}}{2}\left(\xi-\xi_{0}\right)\right)\right)}{ \pm \exp \left( \pm \frac{\sqrt{a \omega}}{2}\left(\xi-\xi_{0}\right)\right)}\right\}^{2}
$$

Family 2.1.3. $\Delta<0$. Then we have $f(v)=a \omega\left((v-\alpha)^{2}+\beta^{2}\right)$, where $\alpha=\frac{2 b}{3 a \omega}, \beta=$ $\sqrt{\frac{b_{1}}{a \omega}-\frac{4 b^{2}}{9 a^{2} \omega^{2}}}$. We get the solution

$$
u=\left\{\alpha \pm \beta \operatorname{coth}\left( \pm \frac{\sqrt{a \omega}}{2}\left(\xi-\xi_{0}\right)\right)\right\}^{2}
$$

Case 2. $b_{0} \neq 0$. Without loss of generality, assume $a \omega>0$ and denote

$$
F(v)=v^{4}+a_{3} v^{3}+a_{2} v^{2}+a_{1} x+a_{0}
$$

where $a_{3}=-\frac{4 b}{3 a \omega}, a_{2}=\frac{b_{1}}{a \omega}, a_{0}=\frac{b_{0}}{a \omega}$. The complete discrimination system for the fourth order polynomial $F(v)$ is given by [11]

$$
\begin{aligned}
& D_{1}=4, \quad D_{2}=-p, \quad D_{3}=8 r p-2 p^{3}-9 q^{2}, \\
& D_{4}=4 p^{4} r-p^{3} q^{2}+36 p r q^{2}-32 r^{2} p^{2}-\frac{27}{4} q^{4}+64 r^{3}, \\
& F_{2}=9 q^{2}-32 p r,
\end{aligned}
$$

where $p=a_{2}, q=\frac{a_{3}^{3}}{8}-\frac{a_{2} a_{3}}{2}, r=a_{0}+\frac{a_{2} a_{3}^{2}}{16}-\frac{3 a_{3}^{4}}{256}$. There are the following cases to be discussed.

Family 2.2.1. $D_{4}=0, D_{3}=0, D_{2}<0$. Then we have

$$
F(v)=v^{2}+l v+s^{2}
$$

where $l, s$ are real numbers, and $l^{2}-4 s^{2}<0$. We have the solution represented by the implicit function $u$ on $\xi$

$$
\begin{aligned}
& \pm \frac{\sqrt{a \omega}}{2}\left(\xi-\xi_{0}\right)=\frac{1}{2 \sqrt{2 s-l}} \ln \frac{\sqrt{u} \mp \sqrt{2 s-l} u^{\frac{1}{4}}+s}{\sqrt{u} \pm \sqrt{2 s-l} u^{\frac{1}{4}}+s} \\
& \quad+\frac{1}{\sqrt{2 s+l}}\left\{\arctan \frac{4 u^{\frac{1}{4}} \pm \sqrt{2 s-l}}{2 \sqrt{2 s+l}}+\arctan \frac{4 u^{\frac{1}{4}} \mp \sqrt{2 s-l}}{2 \sqrt{2 s+l}}\right\} .
\end{aligned}
$$

Family 2.2.2. $D_{4}=0, D_{3}=0, D_{2}=0$. Then we have

$$
F(v)=(v-\alpha)^{4}
$$


If $\alpha>0$, we have

$$
\pm \frac{\sqrt{a \omega}}{2}\left(\xi-\xi_{0}\right)=\frac{1}{2 \sqrt{\alpha}} \ln \left|\frac{u^{\frac{1}{4}}-\sqrt{\alpha}}{u^{\frac{1}{4}}+\sqrt{\alpha}}\right|-\frac{u^{\frac{1}{4}}}{\sqrt{u}-\alpha}
$$

If $\alpha<0$, we have

$$
\pm \frac{\sqrt{a \omega}}{2}\left(\xi-\xi_{0}\right)=\frac{1}{\sqrt{-\alpha}} \arctan \frac{u^{\frac{1}{4}}}{\sqrt{-\alpha}}-\frac{u^{\frac{1}{4}}}{\sqrt{u}-\alpha}
$$

Family 2.2.3. $D_{4}=0, D_{3}=0, D_{2}>0, E_{2}=0$. Then we have

$$
F(v)=(v-\alpha)^{2}(u-\beta)^{2},
$$

where $\alpha, \beta$ are real numbers, and $\alpha>\beta$. If $\alpha>\beta>0$, we have

$$
\pm \frac{\sqrt{a \omega}}{2}(\alpha+\beta)\left(\xi-\xi_{0}\right)=\sqrt{\alpha} \ln \left|\frac{u^{\frac{1}{4}}-\sqrt{\alpha}}{u^{\frac{1}{4}}+\sqrt{\alpha}}\right|-\sqrt{\beta} \ln \left|\frac{u^{\frac{1}{4}}-\sqrt{\beta}}{u^{\frac{1}{4}}+\sqrt{\beta}}\right|
$$

If $0>\alpha>\beta$, we have

$$
\pm \frac{\sqrt{a \omega}}{2}(\alpha+\beta)\left(\xi-\xi_{0}\right)=2 \sqrt{-\alpha} \arctan \frac{u^{\frac{1}{4}}}{\sqrt{-\alpha}}-2 \sqrt{-\beta} \arctan \frac{u^{\frac{1}{4}}}{\sqrt{-\beta}}
$$

If $\alpha>0>\beta$, we have

$$
\pm \frac{\sqrt{a \omega}}{2}(\alpha+\beta)\left(\xi-\xi_{0}\right)=\sqrt{\alpha} \ln \left|\frac{u^{\frac{1}{4}}-\sqrt{\alpha}}{u^{\frac{1}{4}}+\sqrt{\alpha}}\right|-2 \sqrt{-\beta} \arctan \frac{u^{\frac{1}{4}}}{\sqrt{-\beta}}
$$

Family 2.2.4. In all other cases such as $F(v)=(v-\alpha)^{2}(v-\beta)(v-\gamma)$ and so on, the corresponding solutions can be expressed in terms of elliptic functions and hyper-elliptic functions. We omit them for brevity.

\section{Classification of solutions: second case}

Now we consider the second case $\gamma=\frac{3}{2}$. Then we have

$$
\int \frac{v \mathrm{~d} v}{\sqrt{b_{0}+b_{1} v^{2}+a \omega v^{4}-\frac{4 b}{5} v^{5}}}= \pm \frac{1}{2}\left(\xi-\xi_{0}\right) .
$$

By taking the transformation

$$
v=\left(-\frac{5}{4 b}\right)^{\frac{1}{5}} w-\frac{a \omega}{4 b}
$$

the above equation becomes

$$
\int \frac{\left(w-s_{0}\right) \mathrm{d} w}{\sqrt{F(w)}}= \pm\left(-\frac{4 b}{5}\right)^{\frac{2}{5}} \frac{1}{2}\left(\xi-\xi_{0}\right)
$$


where

$$
F(w)=w^{5}+p w^{3}+q w^{2}+r w+s,
$$

and

$$
\begin{aligned}
& p=10 d^{2}-4 c_{4} d, \quad q=-10 d^{3}+6 c_{4} d^{2}+c_{2}, \quad r=5 d^{4}-4 c_{4} d^{3}-2 c_{2} d, \\
& s=b_{0}-d^{5}+c_{4} d^{4}+c_{2} d^{2}, \quad c_{4}=a \omega\left(-\frac{5}{4 b}\right)^{\frac{4}{5}}, \\
& c_{2}=\left(b-\frac{5}{4 b}\right)^{\frac{2}{5}}, \quad s_{0}=\frac{a \omega}{4 b}\left(-\frac{4 b}{5}\right)^{\frac{1}{5}} .
\end{aligned}
$$

We write its complete discrimination system of $F(w)$ as follows (see $[7,10])$ :

$$
\begin{aligned}
D_{2}= & -p, \quad D_{3}=40 r p-12 p^{3}-45 q^{2} \\
D_{4}= & 12 p^{4} r-4 p^{3} q^{2}+117 p r q^{2}-88 r^{2} p^{2} \\
& -40 q s p^{2}-27 q^{4}-300 q r s+160 r^{3} \\
D_{5}= & -1600 q s r^{3}-3750 p q s^{3}+2000 p s^{2} r^{2}-4 p^{3} q^{2} r^{2}+16 p^{3} q^{3} s \\
& -900 r s^{2} p^{3}+825 p^{2} q^{2} s^{2}+144 p q^{2} r^{3}+2250 r q^{2} s^{2}+16 p^{4} r^{3} \\
& +108 p^{5} s^{2}-128 r^{4} p^{2}-27 r^{2} q^{4}+108 s q^{5}+256 r^{5}+3125 s^{4} \\
& -72 r s q p^{4}+560 s q r^{2} p^{2}-630 p r s q^{3}, \\
E_{2}= & 160 r^{2} p^{3}+900 q^{2} r^{2}-48 r p^{5}+60 r P^{2} q^{2}+1500 p q r s+16 q^{2} p^{4} \\
& -1100 q s p^{3}+625 s^{2} p^{2}-3375 s q^{3}, \\
F_{2}= & 3 q^{2}-8 r p .
\end{aligned}
$$

According to the above complete discrimination system, we list the following eleven cases to discuss. Among these, in first five cases, the solutions can be represented in terms of elementary functions, while in other cases the solutions are given by elliptic functions or elliptic integrals.

Family 3.1. $D_{5}=0, D_{4}=0, D_{3}>0, E_{2} \neq 0$. Then we have

$$
F(w)=(w-\alpha)^{2}(w-\beta)^{2}(w-\gamma),
$$

where $\alpha, \beta, \gamma$ are real numbers, and $\gamma \neq \alpha>\beta \neq \gamma$. When $w>\gamma$, we have

$$
\begin{aligned}
\pm\left(\xi-\xi_{0}\right)= & \frac{2\left(\alpha-s_{0}\right)}{(\alpha-\beta) \sqrt{\gamma-\alpha}} \arctan \frac{\sqrt{w-\gamma}}{\sqrt{\gamma-\alpha}} \\
& -\frac{2\left(s_{0}-\beta\right)}{(\alpha-\beta) \sqrt{\gamma-\beta}} \arctan \frac{\sqrt{w-\gamma}}{\sqrt{\gamma-\beta}}, \quad \gamma>\alpha, \\
\pm\left(\xi-\xi_{0}\right)= & -\frac{2\left(s_{0}-\beta\right)}{(\alpha-\beta) \sqrt{\gamma-\beta}} \arctan \frac{\sqrt{\left(\left(-\frac{5}{4 b}\right)^{1 / 5}-\frac{a}{4 b}\right)^{-1} v-\gamma}}{\sqrt{\gamma-\beta}}
\end{aligned}
$$




$$
\begin{aligned}
& +\frac{\left(\alpha-s_{0}\right)}{(\alpha-\beta) \sqrt{\alpha-\gamma}} \\
& \times \ln \left|\frac{\sqrt{\left(\left(-\frac{5}{4 b}\right)^{1 / 5}-\frac{a}{4 b}\right)^{-1} v-\gamma}-\sqrt{\alpha-\gamma}}{\sqrt{\left(\left(-\frac{5}{4 b}\right)^{1 / 5}-\frac{a}{4 b}\right)^{-1} v-\gamma}+\sqrt{\alpha-\gamma}}\right|, \quad \beta<\gamma<\alpha,
\end{aligned}
$$

or

$$
\begin{aligned}
\pm\left(\xi-\xi_{0}\right)= & \frac{\alpha-s_{0}}{(\alpha-\beta) \sqrt{\alpha-\gamma}} \ln \left|\frac{\sqrt{\left(\left(-\frac{5}{4 b}\right)^{1 / 5}-\frac{a}{4 b}\right)^{-1} v-\gamma}-\sqrt{\alpha-\gamma}}{\sqrt{\left(\left(-\frac{5}{4 b}\right)^{1 / 5}-\frac{a}{4 b}\right)^{-1} v-\gamma}+\sqrt{\alpha-\gamma}}\right| \\
& -\frac{s_{0}-\beta}{(\alpha-\beta) \sqrt{\beta-\gamma}} \ln \left|\frac{\sqrt{\left(\left(-\frac{5}{4 b}\right)^{1 / 5}-\frac{a}{4 b}\right)^{-1} v-\gamma}-\sqrt{\beta-\gamma}}{\sqrt{\left(\left(-\frac{5}{4 b}\right)^{1 / 5}-\frac{a}{4 b}\right)^{-1} v-\gamma}+\sqrt{\beta-\gamma}}\right|, \quad \gamma<\beta .
\end{aligned}
$$

Family 3.2. $D_{5}=0, D_{4}=0, D_{3}=0, D_{2} \neq 0, F_{2} \neq 0$. Then we have

$$
F(w)=(w-\alpha)^{3}(w-\beta)^{2},
$$

where $\alpha, \beta$ are real numbers, and $\alpha \neq \beta$. When $w>\alpha$, we have

$$
\begin{aligned}
\pm \frac{\alpha-\beta}{2}\left(\xi-\xi_{0}\right)= & \frac{\beta-s_{0}}{\sqrt{\left(\left(-\frac{5}{4 b}\right)^{1 / 5}-\frac{a}{4 b}\right)^{-1} v-\alpha}} \\
& +\left(\alpha-s_{0}\right) \sqrt{\alpha-\beta} \arctan \frac{\sqrt{\left(\left(-\frac{5}{4 b}\right)^{1 / 5}-\frac{a}{4 b}\right)^{-1} v-\alpha}}{\sqrt{\alpha-\beta}}, \quad \alpha>\beta,
\end{aligned}
$$

or

$$
\begin{aligned}
\pm \frac{\alpha-\beta}{2}\left(\xi-\xi_{0}\right)= & \frac{\beta-s_{0}}{\sqrt{\left(\left(-\frac{5}{4 b}\right)^{1 / 5}-\frac{a}{4 b}\right)^{-1} v-\alpha}} \\
& -\frac{\alpha-s_{0}}{2 \sqrt{\beta-\alpha}} \ln \left|\frac{\sqrt{\left(\left(-\frac{5}{4 b}\right)^{1 / 5}-\frac{a}{4 b}\right)^{-1} v-\alpha}-\sqrt{\beta-\alpha}}{\sqrt{\left(\left(-\frac{5}{4 b}\right)^{1 / 5}-\frac{a}{4 b}\right)^{-1} v-\alpha}+\sqrt{\beta-\alpha}}\right|, \quad \alpha<\beta .
\end{aligned}
$$

Family 3.3. $D_{5}=0, D_{4}=0, D_{3}=0, D_{2} \neq 0, F_{2}=0$. Then we have

$$
F(w)=(w-\alpha)^{4}(w-\beta),
$$

where $\alpha, \beta$, are real numbers, and $\alpha \neq \beta$. When $w>\alpha$, we have

$$
\begin{aligned}
\pm\left(\xi-\xi_{0}\right)= & \frac{\alpha-s_{0}}{\alpha-\beta} \frac{\sqrt{\left(\left(-\frac{5}{4 b}\right)^{1 / 5}-\frac{a}{4 b}\right)^{-1} v-\beta}}{\left(\left(-\frac{5}{4 b}\right)^{1 / 5}-\frac{a}{4 b}\right)^{-1} v-\alpha} \\
& +\left\{\frac{\alpha-s_{0}}{2(\alpha-\beta)^{\frac{3}{2}}}-\frac{1}{\sqrt{\alpha-\beta}}\right\} \arctan \frac{\sqrt{\left(\left(-\frac{5}{4 b}\right)^{1 / 5}-\frac{a}{4 b}\right)^{-1} v-\beta}}{\sqrt{\beta-\alpha}}, \quad \alpha<\beta,
\end{aligned}
$$


or

$$
\begin{aligned}
\pm\left(\xi-\xi_{0}\right)= & \frac{\alpha-s_{0}}{\beta-\alpha} \frac{\sqrt{\left.\left(-\frac{5}{4 b}\right)^{1 / 5}-\frac{a}{4 b}\right)^{-1} v-\beta}}{\left(\left(-\frac{5}{4 b}\right)^{1 / 5}-\frac{a}{4 b}\right)^{-1} v-\alpha} \\
& +\left\{\frac{\alpha-s_{0}}{(\beta-\alpha)^{\frac{3}{2}}}+\frac{1}{\sqrt{\beta-\alpha}}\right\} \\
& \times \ln \left|\frac{\sqrt{\left(\left(-\frac{5}{4 b}\right)^{1 / 5}-\frac{a}{4 b}\right)^{-1} v-\beta}-\sqrt{\beta-\alpha}}{\sqrt{\left(\left(-\frac{5}{4 b}\right)^{1 / 5}-\frac{a}{4 b}\right)^{-1} v-\beta}+\sqrt{\beta-\alpha}}\right|, \quad \alpha>\beta .
\end{aligned}
$$

Family 3.4. $D_{5}=0, D_{4}=0, D_{3}=0, D_{2}=0$. Then we have

$$
F(w)=(w-\alpha)^{5}
$$

where $\alpha$ is real number. When $w>\alpha$, we have

$$
\begin{aligned}
\pm\left(\xi-\xi_{0}\right)= & -2\left(\left(\left(-\frac{5}{4 b}\right)^{1 / 5}-\frac{a}{4 b}\right)^{-1} v-\alpha\right)^{-\frac{1}{2}} \\
& -\frac{2\left(\alpha-s_{0}\right)}{3}\left(\left(\left(-\frac{5}{4 b}\right)^{1 / 5}-\frac{a}{4 b}\right)^{-1} v-\alpha\right)^{-\frac{2}{3}}
\end{aligned}
$$

Family 3.5. $D_{5}=0, D_{4}=0, D_{3}<0, E_{2} \neq 0$. Then we have

$$
F(w)=(w-\alpha)\left(w^{2}+r w+s\right)^{2},
$$

where $\alpha$ is real number, and $r^{2}-4 s<0$. When $w>\alpha$, we have

$$
\begin{aligned}
\pm\left(\xi-\xi_{0}\right)= & \frac{b-\alpha+s_{0}}{4 a b} \ln \left(\left(\left(-\frac{5}{4 b}\right)^{1 / 5}-\frac{a}{4 b}\right)^{-2}\left(\left(-\frac{5}{4 b}\right)^{1 / 5}-\frac{a}{4 b}\right)^{-2} v^{2}\right. \\
& \left.+r\left(\left(-\frac{5}{4 b}\right)^{1 / 5}-\frac{a}{4 b}\right)^{-1} v+s\right) \\
& +\frac{7 b-\alpha+s_{0}}{2 b \sqrt{4 b-a^{2}}} \arctan \frac{\left.2\left(-\frac{5}{4 b}\right)^{1 / 5}-\frac{a}{4 b}\right)^{-1} v-a}{4 b-a^{2}}
\end{aligned}
$$

where

$$
b=\sqrt{\alpha^{2}+r \alpha+s}, \quad a=\sqrt{2 b-r-2 \alpha} .
$$

Family 3.6. $D_{5}=0, D_{4}>0$. Then we have

$$
F(w)=(w-\alpha)^{2}\left(w-\alpha_{1}\right)\left(w-\alpha_{2}\right)\left(w-\alpha_{3}\right),
$$

where $\alpha, \alpha_{1}, \alpha_{2}, \alpha_{3}$ are real numbers, and $\alpha_{1}>\alpha_{2}>\alpha_{3}$. We have

$$
\begin{aligned}
\pm\left(\xi-\xi_{0}\right)= & \int \frac{\mathrm{d} w}{\sqrt{\left(w-\alpha_{1}\right)\left(w-\alpha_{2}\right)\left(w-\alpha_{3}\right)}} \\
& -\frac{2\left(\alpha-s_{0}\right)}{\left(\alpha-\alpha_{2}\right) \sqrt{\alpha_{2}-\alpha_{3}}}\left\{\mathrm{~F}(\varphi, k)-\frac{\alpha_{1}-\alpha_{2}}{\alpha_{1}-\alpha} \Pi\left(\varphi, \frac{\alpha_{1}-\alpha_{2}}{\alpha_{1}-\alpha}, k\right)\right\},
\end{aligned}
$$


where

$$
\begin{aligned}
& \mathrm{F}(\varphi, k)=\int_{0}^{\varphi} \frac{\mathrm{d} \varphi}{\sqrt{1-k^{2} \sin ^{2} \varphi}}, \\
& \Pi(\varphi, h, k)=\int_{0}^{\varphi} \frac{\mathrm{d} \varphi}{\left(1+h \sin ^{2} \varphi\right) \sqrt{1-k^{2} \sin ^{2} \varphi}} .
\end{aligned}
$$

Family 3.7. $D_{5}=0, D_{4}=0, D_{3}<0, E_{2}=0$. Then we have

$$
F(w)=(w-\alpha)^{3}\left(\left(w-l_{1}\right)^{2}+s_{1}^{2}\right),
$$

where $\alpha, l_{1}$, and $s_{1}$ are real numbers. When $w>\alpha$ and $\alpha \neq l_{1}+s_{1}$, we have

$$
\begin{aligned}
\pm\left(\xi-\xi_{0}\right)= & \int \frac{\mathrm{d} w}{\sqrt{(w-\alpha)\left(\left(w-l_{1}\right)^{2}+s_{1}^{2}\right)}} \\
& +\left(\alpha-s_{0}\right)\left\{\frac{\tan \theta+\cot \theta}{2\left(s_{1} \tan \theta-l_{1}-\alpha\right) \sqrt{\frac{s_{1}}{\sin ^{3} 2 \theta}}} \mathrm{F}(\varphi, k)-\frac{s_{1} \tan \theta+s_{1} \cot \theta}{s_{1} \cot \theta+l_{1}+\alpha}\right. \\
& \times\left\{\left(\frac{\tan \theta+l_{1}+\alpha}{\left(s_{1} \cot \theta+l_{1}-\alpha\right) \sin \varphi} \sqrt{1-k^{2} \sin ^{2} \varphi}+\mathrm{F}(\varphi, k)-\mathrm{E}(\varphi, k)\right\}\right\},
\end{aligned}
$$

where

$$
E(\varphi, k)=\int_{0}^{\varphi} \sqrt{1-k^{2} \sin ^{\theta}} \mathrm{d} \theta .
$$

Family 3.8. $D_{5}=0, D_{4}<0$. Then we have

$$
F(w)=(w-\alpha)^{2}(w-\beta)\left(\left(w-l_{1}\right)^{2}+s_{1}^{2}\right),
$$

where $\alpha, l_{1}$, and $s_{1}$ are real numbers. The solution is represented by

$$
\begin{aligned}
\pm\left(\xi-\xi_{0}\right)= & \int \frac{\mathrm{d} w}{\sqrt{(w-\beta)\left(\left(w-l_{1}\right)^{2}+s_{1}^{2}\right)}} \\
& +\left(\alpha-s_{0}\right)\left\{\frac{\tan \theta+\cot \theta}{2\left(s_{1} \tan \theta-l_{1}-\alpha\right) \sqrt{\frac{s}{\sin ^{3} 2 \theta}}} \mathrm{F}(\varphi, k)-\frac{s_{1} \tan \theta+s \cot \theta}{s_{1} \cot \theta+l_{1}+\alpha}\right. \\
& \times\left\{\left(\frac{\tan \theta+l_{1}+\alpha}{\left(s \cot \theta+l_{1}-\alpha\right) \sin \varphi} \sqrt{1-k^{2} \sin ^{2} \varphi}+\mathrm{F}(\varphi, k)-\mathrm{E}(\varphi, k)\right\}\right\} .
\end{aligned}
$$

Family 3.9. $D_{5}=0, D_{4}=0, D_{3}>0, E_{2}=0$. Then we have

$$
F(w)=(w-\alpha)^{3}(w-\beta)(w-\gamma),
$$

where $\alpha, \beta$, and $\gamma$ are real numbers. The solution is represented by

$$
\begin{aligned}
\pm\left(\xi-\xi_{0}\right)= & \int \frac{\mathrm{d} w}{\sqrt{(w-\alpha)(w-\beta)(w-\gamma)}} \\
& +\frac{(\alpha-\beta)\left(\alpha-s_{0}\right)}{2 \sqrt{\alpha-\gamma}} \mathrm{E}\left(\arcsin \sqrt{\frac{\alpha-\gamma}{w-\gamma}}, \sqrt{\frac{\beta-\gamma}{\alpha-\gamma}}\right)-\sqrt{\frac{w-\beta}{(w-\gamma)(w-\alpha)}} .
\end{aligned}
$$


In other cases, we can give the corresponding solutions similarly. We omit them for simplicity.

Family 3.10. In the following three cases: $D_{5}>0, D_{4}>0, D_{3}>0, D_{2}>0$ or $D_{5}<0$ or $D_{5}>$ $0 \wedge\left(D_{4} \leq 0 \vee D_{3} \leq 0 \vee D_{2} \leq 0\right)$, where $\wedge$ means "and", $\vee$ means "or", we have respectively

$$
\begin{aligned}
& F(w)=\left(w-\alpha_{1}\right)\left(w-\alpha_{2}\right)\left(w-\alpha_{3}\right)\left(w-\alpha_{4}\right)\left(w-\alpha_{5}\right), \\
& F(w)=\left(w-\alpha_{1}\right)\left(w-\alpha_{2}\right)\left(w-\alpha_{3}\right)\left((w-l)^{2}+s^{2}\right),
\end{aligned}
$$

or

$$
\left.F(w)=(w-\alpha)\left(\left(w-l_{1}\right)^{2}+s_{1}^{2}\right)\right)\left(\left(w-l_{2}\right)^{2}+s_{2}^{2}\right) .
$$

Then the corresponding solutions can be expressed by hyper-elliptic functions or hyperelliptic integral. We omit them for brevity.

\section{Conclusion}

By the complete discrimination system for polynomial method, we give the complete classification of the traveling wave solutions to a fractional power ZK equation under $\gamma=\frac{1}{2}$ and $\gamma=\frac{3}{2}$. The last case is more difficult than the first one since we need the complete discrimination system for fifth order polynomial. These results mean that there are rich traveling wave patterns for the ZK equation. Our results provide a complete classification of all single traveling wave solutions to two fractional power ZK equations. If we take the concrete parameters in a real model, we can give the corresponding representation of the solution. Therefore, it is rather convenient for practice. On the other hand, most of the solutions have implicit function forms if we consider $w$ or $v$ as the function of $\xi$. But, inversely, if we take $\xi$ as the function of $w$ or $v$, the solutions will become explicit functions. In general, these implicit function solutions cannot be obtained by the direct expansion methods and ansatz methods in [46, 47].

Acknowledgements

The authors would like to thank to reviewers for their helpful suggestions.

Funding

This project is supported by the National Natural Science Foundation of China under Grant No. 51674086, 51534004.

Competing interests

We would like to declare no conflicts of interest.

Authors' contributions

YL gave the main ideas and computations including those in Sect. 1, Sect. 3, and Sect. 4. XW gave the computation of Sect. 2. Both authors read and approved the final manuscript.

\section{Publisher's Note}

Springer Nature remains neutral with regard to jurisdictional claims in published maps and institutional affiliations.

Received: 1 January 2019 Accepted: 13 March 2019 Published online: 08 April 2019

\section{References}

1. En-Gui, F.: Integrable Systems and Computer Algebra. Academic Press, Bejing (2004)

2. Hirota, R.: The Direct Method in Soliton Theory. Cambridge University Press, Cambridge (2004)

3. Liu, C.: Canonical-like transformation method and exact solutions to a class of diffusion equations. Chaos Solitons Fractals 42(1), 441-446 (2009)

4. Ma, W.X., Zhu, Z.: Solving the $(3+1)$-dimensional generalized KP and BKP equations by the multiple exp-function algorithm. Appl. Math. Comput. 218(24), 11871-11879 (2012) 
5. Wazwaz, A.M.: Partial Differential Equations and Solitary Waves Theory. Springer, Berlin (2010)

6. Liu, C.: How many first integrals imply integrability in infinite-dimensional Hamilton system. Rep. Math. Phys. 67(1), 109-123 (2011)

7. Liu, C.: Travelling wave solutions of triple sine-Gordon equation. Chin. Phys. Lett. 21(12), 2369-2371 (2004)

8. Liu, C.: New exact envelope traveling wave solutions to higher-order dispersive cubic-qintic nonlinear Schrödinger equation. Commun. Theor. Phys. 44(5), 799-801 (2005)

9. Liu, C.: Traveling wave solutions of a kind of generalized Ginzburg-Landau equation. Commun. Theor. Phys. 43(4), 787-790 (2005)

10. Liu, C: Solution of ODE $u^{\prime \prime}+p(u)\left(u^{\prime}\right)^{2}+q(u)=0$ and applications to classifications of all single travelling wave solutions to some nonlinear mathematical physics equations. Commun. Theor. Phys. 49(2), 291-296 (2008)

11. Liu, C.: Applications of complete discrimination system for polynomial for classifications of traveling wave solutions to nonlinear differential equations. Comput. Phys. Commun. 181(2), 317-324 (2010)

12. Liu, C.: Trial equation method and its applications to nonlinear evolution equations. Acta Phys. Sin. 54(6), 2505-2509 (2005)

13. Liu, C.: Using trial equation method to solve the exact solutions for two kinds of KdV equations with variable coefficients. Acta Phys. Sin. 54(10), 4506-4510 (2005)

14. Liu, C.: Trial equation method for nonlinear evolution equations with rank inhomogeneous: mathematical discussions and applications. Commun. Theor. Phys. 45(2), 219-223 (2006)

15. Liu, C.: Trial equation method based on symmetry and applications to nonlinear equations arising in mathematical physics. Found. Phys. 41(5), 793-804 (2011)

16. Liu, C.: Exponential function rational expansion method for nonlinear differential-difference equations. Chaos Solitons Fractals 40, 708-716 (2009)

17. Liu, Y:: Exact solutions to nonlinear Schrodinger equation with variable coefficients. Appl. Math. Comput. 217(12), 5866-5869 (2011)

18. Xin, W., Yang, L.: All envelop traveling wave patterns to nonlinear Schrodinger equation in parabolic law medium. Mod. Phys. Lett. B 33(01), 1850428 (2019)

19. Xin, W., Yang, L.: All single travelling wave patterns to fractional Jimbo-Miwa equation and Zakharov-Kuznetsov equation. Pramāna 92(3), 31 (2019)

20. Cheng, Y:: Classification of traveling wave solutions to the Vakhnenko equations. Comput. Math. Appl. 62(10), 3987-3996 (2011)

21. Yang, S.: The envelope travelling wave solutions to the Gerdjikov-Ivanov model. Pramāna 91(3), 36 (2018)

22. Pandir, Y., Gurefe, Y., Misirli, E.: Classification of exact solutions to the generalized Kadomtsev-Petviashvili equation. Phys. Scr. 87(2), 025003 (2013)

23. Chen, S.Q., Liu, Y., Wei, L.X., Guan, B.: Exact solutions to fractional Drinfeld-Sokolov-Wilson equations. Chin. J. Phys. 56(2), 708-720 (2018)

24. Seadawy, A.R.: Nonlinear wave solutions of the three dimensional Zakharov-Kuznetsov-Burgers equation in dusty plasma. Phys. A, Stat. Mech. Appl. 439, 124-131 (2015)

25. Khater, A.H., Seadawy, A.R., Helal, M.A.: General soliton solutions of an n-dimensional nonlinear Schrodinger equation. Nuovo Cimento B 115(11), 1303-1311 (2000)

26. Seadawy, A.R.: The generalized nonlinear higher order of KdV equations from the higher order nonlinear Schrodinger equation and its solutions. Optik 139, 31-43 (2017)

27. Khater, A.H., Callebaut, D.K., Seadawy, A.R.: General soliton solutions of an n-dimensional complex Ginzburg-Landau equation. Phys. Scr. 62(5), 353-357 (2000)

28. Seadawy, A.R:: Modulation instability analysis for the generalized derivative higher order nonlinear Schrodinger equation and its the bright and dark soliton solutions. J. Electromagn. Waves Appl. 31(14), 1353-1362 (2017)

29. Khater, A.H., Callebaut, D.K., Seadawy, A.R.: Nonlinear dispersive instabilities in Kelvin-Helmholtz magnetohydrodynamic flows. Phys. Scr. 67(4), 340-349 (2003)

30. Arshad, M., Seadawy, A.R., Lu, D.: Exact bright-dark solitary wave solutions of the higher-order cubic-quintic nonlinear Schrodinger equation and its stability. Optik 138, 40-49 (2017)

31. Seadawy, A.R.: Ion acoustic solitary wave solutions of two-dimensional nonlinear Kadomtsev-Petviashvili-Burgers equation in quantum plasma. Math. Methods Appl. Sci. 40(5), 1598-1607 (2017)

32. Helal, M.A., Seadawy, A.R.: Variational method for the derivative nonlinear Schrodinger equation with computational applications. Phys. Scr. 80(3), 035004 (2009)

33. Helal, M.A., Seadawy, A.R.: Exact soliton solutions of a D-dimensional nonlinear Schrodinger equation with damping and diffusive terms. Z. Angew. Math. Phys. 62(5), 839-847 (2011)

34. Ma, W.X., Zhou, Y.: Lump solutions to nonlinear partial differential equations via Hirota bilinear forms. J. Differ. Equ. 264(4), 2633-2659 (2018)

35. Ma, W.X., Li, J., Khalique, C.M.: A study on lump solutions to a generalized Hirota-Satsuma-Ito equation in (2+1)-dimensions. Complexity 2018, Article ID 9059858 (2018)

36. Ma, W.X., Yong, X., Zhang, H.Q.: Diversity of interaction solutions to the $(2+1)$-dimensional Ito equation. Comput. Math. Appl. 75(1), 289-295 (2018)

37. Yang, J.Y., Ma, W.X., Qin, Z.: Lump and lump-soliton solutions to the $3+1$-dimensional Ito equation. Anal. Math. Phys. $8(3), 427-436(2018)$

38. Ma, W.X.: Abundant lumps and their interaction solutions of (3+1)-dimensional linear PDEs. J. Geom. Phys. 133, $10-16(2018)$

39. Ma, W.X.: Lump and interaction solutions of linear PDEs in (3 + 1)-dimensions. East Asian J. Appl. Math. 9(1), 185-194 (2019)

40. Yang, J.Y., Ma, W.X., Qin, Z.Y.: Abundant mixed lump-soliton solutions to the BKP equation. East Asian J. Appl. Math. 8(2), 224-232 (2018)

41. Zhou, Y., Manukure, S., Ma, W.X.: Lump and lump-soliton solutions to the Hirota-Satsuma-Ito equation. Commun. Nonlinear Sci. Numer. Simul. 68, 56-62 (2019)

42. Zakharov, V.E., Kuznetsov, E.A.: On three-dimensional solitons. Sov. Phys. 39, 285-288 (1974) 
43. Monro, S., Parkes, E.J.: The derivation of a modified Zakharov-Kuznetsov equation and the stability of its solutions J. Plasma Phys. 62(3), 305-317 (1999)

44. Monro, S., Parkes, E.J.: Stability of solitary-wave solutions to a modified Zakharov-Kuznetsov equation. J. Plasma Phys. 64(3), 411-426 (2000)

45. Schamel, H.: A modified Korteweg-de-Vries equation for ion acoustic waves due to resonant electrons. J. Plasma Phys. 9(3), 377-387 (1973)

46. Wazwaz, A.M.: Exact solutions with solitons and periodic structures for the Zakharov-Kuznetsov (ZK) equation and its modified form. Commun. Nonlinear Sci. Numer. Simul. 10, 597-606 (2005)

47. Li, B., Chen, Y., Zhang, H.: Exact travelling wave solutions for a generalized Zakharov-Kuznetsov equation. Appl. Math. Comput. 146, 653-666 (2003)

48. Shivamoggi, B.K.: The Painleve analysis of the Zakharov-Kuznetsov equation. Phys. Scr. 42, 641-642 (1990)

Submit your manuscript to a SpringerOpen ${ }^{\circ}$ journal and benefit from:

- Convenient online submission

- Rigorous peer review

- Open access: articles freely available online

- High visibility within the field

- Retaining the copyright to your article

Submit your next manuscript at $\gg$ springeropen.com 